\title{
Obesidade Visceral e Hipertensão Sistólica como Substratos da Disfunção Endotelial em Adolescentes Obesos
}

\author{
Visceral Obesity and High Systolic Blood Pressure as the Substrate of Endothelial Dysfunction in Obese Adolescents \\ Maria Fernanda Hussid, ${ }^{1}{ }^{\circledR 0}$ Felipe Xerez Cepeda, ${ }^{1}{ }^{\circledR}$ Camila P. Jordão, ${ }^{2}$ Rafaela R. P. Lopes-Vicente, ${ }^{1}$ Leslie \\ Virmondes, ${ }^{1}$ Keyla Y. Katayama, ${ }^{1}$ Ezequiel F. de Oliveira, ${ }^{1}$ Luis V. F. Oliveira, ${ }^{3}{ }^{\circledR}$ Fernanda Marciano Consolim- \\ Colombo, ${ }^{1,2}$ Ivani Credidio Trombetta ${ }^{1,2}$ \\ Universidade Nove de Julho (UNINOVE), ${ }^{1}$ São Paulo, SP - Brasil \\ Instituto do Coração (InCor), Hospital das Clínicas da Faculdade de Medicina da Universidade de São Paulo, ${ }^{2}$ São Paulo, SP - Brasil \\ Centro Universitário de Anápolis (UniEvangélica), ${ }^{3}$ Anápolis, GO - Brasil
}

\section{Resumo}

Fundamento: A obesidade afeta a adolescência, podendo levar à síndrome metabólica (SM) e disfunção endotelial, um marcador precoce de risco cardiovascular. Apesar de a obesidade ser fortemente associada à síndrome da apneia obstrutiva do sono (SAOS), ainda não está claro o papel da SAOS na função endotelial em adolescentes obesos.

Objetivo: Investigar se a obesidade durante a adolescência leva à SM e/ou SAOS e causa disfunção endotelial nesses indivíduos. Além disso, estudamos a possível associação dos fatores de risco para SM e do índice de apneia e hipopneia (IAH) com disfunção endotelial.

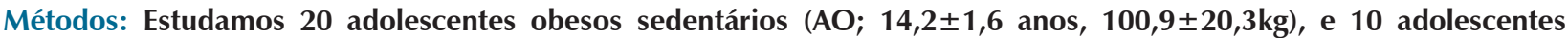
eutróficos $(A E, 15,2 \pm 1,2$ anos, 54,4 $45,3 \mathrm{~kg}$ ) pareados por sexo. Avaliamos os fatores de risco para $\mathrm{SM}$ (critérios da Federação Internacional de Diabetes), função vascular (dilatação mediada pelo fluxo, DMF), capacidade funcional (VO $\mathrm{V}_{2}$ ico) e presença de SAOS (IAH $>1$ evento/hora, pela polissonografia). Consideramos um $\mathrm{p}<0,05$ como estatisticamente significativo.

Resultados: AO apresentaram maior circunferência da cintura (CC), gordura corporal, triglicerídeos, pressão arterial sistólica (PAS) e diastólica (PAD), maiores níveis de LDL e menores $\mathrm{HDL}$ e VO $\mathrm{P}_{2}$ pico em comparação a AE. Não houve diferença no IAH entre os grupos. AO apresentaram menor DMF que $A E(6,17 \pm 2,72$ vs. 9,37 $\pm 2,20 \%, p=0,005)$. Observou-se uma associação entre DMF e CC $(R=-0,506, p=0,008)$ e entre DMF e PAS $(R=-0,493, p=0,006)$.

Conclusão: Em adolescentes, a obesidade associou-se à SM e causou disfunção endotelial. CC e PAS aumentadas poderiam estar envolvidas nessa alteração. SAOS foi detectada na maioria dos adolescentes independentemente de obesidade. (Arq Bras Cardiol. 2021; 116(4):795-803)

Palavras-chave: Adolescente; Obesidade; Síndrome Metabólica; Hipertensão; Diabetes; Circunferência da Cintura, Síndrome da Apneia Obstrutiva do Sono, Endotélio, Fatores de Risco.

\section{Abstract}

Background: Obesity affects adolescence and may lead to metabolic syndrome (MetS) and endothelial dysfunction, an early marker of cardiovascular risk. Albeit obesity is strongly associated with obstructive sleep apnea (OSA), it is not clear the role of OSA in endothelial function in adolescents with obesity.

Objective: To investigate whether obesity during adolescence leads to MetS and/or OSA; and causes endothelial dysfunction. In addition, we studied the possible association of MetS risk factors and apnea hypopnea index (AHI) with endothelial dysfunction.

Methods: We studied 20 sedentary obese adolescents (OA; $14.2 \pm 1.6$ years, $100.9 \pm 20.3 \mathrm{~kg}$ ), and 10 normal-weight adolescents (NWA, $15.2 \pm 1.2$ years, $54.4 \pm 5.3 \mathrm{~kg}$ ) paired for sex. We assessed MetS risk factors (International Diabetes Federation criteria), vascular function (FlowMediated Dilation, FMD), functional capacity ( $\mathrm{O}_{2}$ peak) and the presence of OSA (AHI>1event/h, by polysomnography). We considered statistically significant a $P<0.05$.

Results: OA presented higher waist (WC), body fat, triglycerides, systolic (SBP) and diastolic blood pressure (DBP), LDL-C and lower HDL-C and VO ${ }_{2}$ peak than NWA. MetS was presented in the 35\% of OA, whereas OSA was present in $86.6 \%$ of OA and 50\% of EA. There was no difference

Correspondência: Ivani Credidio Trombetta •

Universidade Nove de Julho (UNINOVE) - Rua Vergueiro, 235/249. CEP 01504-001, São Paulo, SP - Brasil

E-mail: ivani.trombetta@gmail.com

Artigo recebido em 12/08/2019, revisado em 11/11/2019, aceito em 27/12/2019

DOI: https://doi.org/10.36660/abc.20190541 


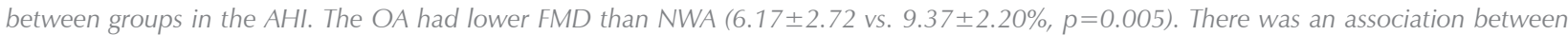
FMD and $W C(R=-0.506, p=0.008)$ and FMD and SBP $(R=-0.493, p=0.006)$

Conclusion: In adolescents, obesity was associates with MetS and caused endothelial dysfunction. Increased WC and SBP could be involved in this alteration. OSA was observed in most adolescents, regardless of obesity. (Arq Bras Cardiol. 2021; 116(4): 795-803)

Keywords: Adolescent; Obesity; Metabolic Syndrome; Hypertension; Diabetes; Waist Circumference; Sleep Apnea Obstructive; Endothelium; Risk Factors.

Full texts in English - http://www.arquivosonline.com.br

\section{Introdução}

A obesidade tem aumentado rapidamente em todo o mundo e é considerada um fator de risco para doenças crônicas não transmissíveis. Crianças e adolescentes têm sido seriamente impactados por essa tendência, particularmente em países em desenvolvimento, segundo a Organização Mundial de Saúde (OMS). ${ }^{1}$ Ao avaliar o estado nutricional de escolares com idade entre 13 e 17 anos por meio do índice de massa corporal (IMC), observa-se que $23,7 \%$ da população do sexo masculino encontra-se com sobrepeso, e 8,3\% são obesos. ${ }^{2} \mathrm{~A}$ preocupação da elevada prevalência de obesidade em crianças e adolescentes baseia-se no fato dessa condição ser um possível preditor de obesidade na fase adulta, levando a um risco aumentado de doenças crônicas, tais como diabetes tipo 2, síndrome metabólica (SM) e doenças cardiovasculares (DCV). ${ }^{3}$

De fato, está claro na literatura que a obesidade está positivamente associada com a incidência de SM. ${ }^{4}$ Estudos realizados com adolescentes na puberdade mostraram uma prevalência de SM variando de 25 a 30\%. ${ }^{5}$ Nesse estudo, os autores encontraram que a circunferência da cintura (CC) foi um preditor de SM, com um aumento de $11 \%$ no risco de SM a cada $1 \mathrm{~cm}$ de incremento na circunferência abdominal. ${ }^{5}$

Já está bem estabelecido que o marcador mais precoce de aterosclerose é disfunção endotelial, ${ }^{6}$ a qual pode ser encontrada tanto na hipertensão como na aterosclerose. A disfunção endotelial também está envolvida em processos fisiológicos e patológicos, incluindo inflamação, resistência insulínica e obesidade, entre outras doenças. ${ }^{6}$

A dilatação mediada pelo fluxo (DMF) na ultrassonografia é um método amplamente utilizado para avaliar função endotelial, o que pode ser um preditor de eventos cardiovasculares tanto em indivíduos assintomáticos como em indivíduos com DCV. Uma mudança na DMF pode ter valor prognóstico em humanos. ${ }^{7}$

As doenças respiratórias do sono estão entre as consequências da obesidade, incluindo a síndrome da apneia obstrutiva do sono (SAOS). A SAOS é o distúrbio respiratório do sono mais comum, com uma prevalência de 1 a $4 \%$ na infância, com pico na faixa etária de 2 a 8 anos. ${ }^{8}$ Em crianças obesas, essa porcentagem pode atingir 36\%. ${ }^{9}$ A SAOS tem sido correlacionada com obesidade, promovendo um ambiente de inflamação moderada e crônica. Pacientes com SAOS apresentam episódios de hipóxia e despertares recorrentes durante o sono devido à atividade aumentada do sistema nervoso simpático. ${ }^{9}$ Trombetta et al., ${ }^{10}$ encontraram que pacientes com SM e SAOS apresentavam maiores níveis de pressão arterial em comparação àqueles com SM e sem SAOS. Atividade simpática aumentada e disfunção do barorreflexo foram observadas nesses pacientes com SAOS associada à SM. ${ }^{10} \mathrm{~A}$ associação entre obesidade e SAOS poderia aumentar o risco de disfunção endotelial. ${ }^{11}$

No presente estudo, adolescentes obesos foram comparados com adolescentes eutróficos quanto à antropometria, composição corporal, parâmetros bioquímicos e reatividade vascular, e apneia do sono. Nosso objetivo foi investigar se a obesidade durante a adolescência: 1) leva à SM e/ou SAOS; e 2) causa disfunção endotelial. Além disso, estudamos a possível associação da SM ou o índice de apneia-hipopneia (IAH) com a disfunção endotelial.

\section{Métodos}

\section{Comitê de Ética}

O estudo foi aprovado pelo comitê de ética em pesquisa da Universidade Nove de Julho (UNINOVE) sob número 973.013, CAAE: 41899215.0.0000.5511. Os pais ou responsáveis dos adolescentes foram informados sobre os procedimentos do estudo e deram consentimento informado por escrito. Os adolescentes também foram informados sobre todos os procedimentos e deram consentimento por escrito.

\section{Sujeitos}

Este foi um estudo transversal. Adolescentes com idade entre 12 e 17 anos atendidos no ambulatório da UNINOVE foram convidados para participarem no estudo de acordo com os critérios de inclusão e de exclusão. Incluímos no estudo adolescentes pós-púberes segundo classificação de Tanner (M4 para meninas ou menarca, e G4 para meninos), ${ }^{12,13}$ eutróficos ou obesos, sedentários, sem tratamento dietético ou medicamentoso para obesidade, com ou sem SM. Os critérios de exclusão foram adolescentes que não se encontravam no estágio póspúbere, com sobrepeso, e aqueles com suspeita ou diagnóstico confirmado de alguma síndrome genética ou distúrbio neuroendócrino, tais como hipotiroidismo descontrolado e diabetes tipo 1. Pacientes com distúrbios alimentares (anorexia nervosa, bulimia nervosa, ou distúrbio alimentar não específico) também foram excluídos. Um total de 20 adolescentes obesos (AO) e 10 adolescentes eutróficos (AE) foram estudados.

O diagnóstico de SM foi estabelecido utilizando os critérios da Federação Internacional de Diabetes (IDF, International Diabetes Federation). Obesidade central foi definida como $\mathrm{CC} \geq 94 \mathrm{~cm}$ para homens e $\geq 80 \mathrm{~cm}$ para mulheres), combinada com dois destes quatro critérios diagnósticos: (1) lipoproteína de alta densidade $(\mathrm{HDL})<40 \mathrm{mg} / \mathrm{dL}(<1,03 \mathrm{mmol} / \mathrm{L})$ em homens e $<50 \mathrm{mg} / \mathrm{dL}(1,29 \mathrm{mmol} / \mathrm{L})$ em mulheres; (2) glicemia 
de jejum $\geq 100 \mathrm{mg} / \mathrm{dL}$ ( $\geq 5,6 \mathrm{mmol} / \mathrm{L}$ ); (3) triglicerídeos de jejum (TG) $\geq 150 \mathrm{mg} / \mathrm{dL}$ (>1,69 mmol/L); e (4) pressão arterial sistólica (PAS) $\geq 130$ e diastólica (PAD) $\geq 85 \mathrm{mmHg} \cdot{ }^{14,15}$

\section{Medidas}

\section{Medidas Antropométricas e Composição Corporal}

Foram avaliados peso e altura, e em seguida calculado o IMC. O IMC foi expresso em escore padrão (escore-z); peso normal foi definido como um z-score entre -2 e +1 ; sobrepeso como um z-score entre +1 e +2 ; e obesidade como $>+2$. A avaliação da composição corporal foi realizada por análise de bioimpedância elétrica (RJL, Quantum II model, Clinton Twp, Mi, EUA). A CC e a circunferência do pescoço (CP) foram medidas conforme descrito anteriormente. ${ }^{16,17}$

\section{Pressão Arterial}

PAS e PAD foram medidas utilizando-se manguito de tamanho apropriado. ${ }^{18-20}$

\section{Análise Sérica}

As amostras de sangue foram coletadas pela manhã após 12 horas de jejum. Foram determinadas concentrações de glicose, TG, colesterol total, HDL-colesterol, lipoproteína de baixa densidade (LDL-colesterol), razão TG/HDL-c e razão LDL-c/HDL-c.

\section{Polissonografia Noturna}

Foi realizada polissonografia de noite inteira (monitoramento padrão - nível 1) utilizando-se um sistema de análise ambulatorial do sono (Embla Somnologica Studio - EMBLA A10, versão 3.1.2.; Flagahf Medical Devices, Iceland), conforme descrito anteriormente. ${ }^{21,22}$ Uma vez que havia adolescentes com idade de 12 anos no estudo, escolhemos os critérios de classificação da AASM (American Academy of Sleep Medicine) para SAOS em crianças. ${ }^{23}$ SAOS foi definida como um IAH $>1$ evento/hora; um IAH $\geq 1$ 4,99 foi considerado SAOS leve; um IAH de 5 a 9,99 foi considerado SAOS moderado, e um IAH $\geq 10$ SAOS grave. ${ }^{9}$

Em crianças, uma apneia é pontuada quando o pico do sinal cai $\geq 90 \%$ da linha de base pré-evento. A hipoventilação é pontuada quando o $\mathrm{CO}_{2}$ arterial (ou substituto) é> $50 \mathrm{~mm} \mathrm{Hg}$ por> 25\% do tempo total de sono. O IAH é a soma do número total de eventos respiratórios (apneias mais hipopneias) por hora de sono. O índice de despertar foi definido como o número médio de despertares por hora de sono. A dessaturação de oxigênio (nadir de $\mathrm{SaO}_{2}$ ) foi definida como a menor saturação de oxigênio da hemoglobina registrada por oximetria de pulso. ${ }^{23}$

\section{Teste de Esforço Cardiopulmonar (TECP)}

O TECP foi realizado em uma esteira conectada a um sistema composto de um módulo de análise de gás, acoplado a um analisador de onda/módulo de fluxo, com medida a cada respiração (breath-by-breath mode) (BreezeCardiO2 System microcomputer; Medical Graphics Corporation-MGC, St. Paul, Mo, EUA), com protocolo de rampa. O TEPC permite medir a capacidade funcional $\left(\mathrm{VO}_{2}\right.$ pico $)$ como o $\mathrm{VO}_{2}$ máximo alcançado ao final do teste. ${ }^{24,25}$

\section{Hiperemia Reativa}

\section{Dilatação Mediada pelo Fluxo (DMF)}

A DMF foi realizada por ultrassom vascular de alta resolução (Vivid i, GE Medical Systems, Tirat Carmel, Israel), medindo-se a dilatação do vaso (dilatação dependente do endotélio) da artéria braquial, como descrito anteriormente. ${ }^{26}$ Em resumo, os participantes deitaram-se em repouso por pelo menos 10 minutos, e o primeiro registro em repouso foi realizado. Em seguida, um fluxo aumentado foi induzido utilizando um esfigmomanômetro, com manguito colocado distalmente à artéria braquial no antebraço, insuflado até uma pressão superior à pressão sistólica (aproximadamente 20-30 $\mathrm{mmHg}$ ) durante 5 minutos. O manguito foi desinflado, e foram registrados o fluxo e a dilatação do vaso, representada pela tensão de cisalhamento. A diferença entre o diâmetro basal e o diâmetro após a dilatação foi avaliada.

Índice de Hiperemia Reativa (IHR) por Tonometria Arterial Periférica

A função endotelial foi avaliada pela medida do IHR por tonometria arterial periférica (Endo-PAT2000; Itamar Medical, Cesareia, Israel) conforme descrito anteriormente. ${ }^{27}$ Esse método avalia a função endotelial microvascular. ${ }^{28}$

Para avaliação tanto da DMF como do IHR, os adolescentes foram orientados a fazerem jejum de 4 a 6 horas, e evitarem consumo de cafeína, chocolate, alimentos gordurosos, e praticarem exercício no dia do exame.

\section{Análise Estatística}

A análise estatística foi realizada usando o programa SPSS20 Statistics (IBM Corp., Armonk, NY, EUA). O tamanho da amostra foi calculado pelo website http://www.openepi. com. Levamos em consideração um poder de $80 \%$, com erro tipo 1 de 0,05 (bicaudal). Usamos variáveis da função endotelial (IHR e DMF) como desfecho primário. Nós escolhemos o maior número de indivíduos, 30 adolescentes para o estudo. A normalidade das amostras foi testada pelo teste de Kolmogorov-Smirnov. As variáveis paramétricas foram expressas em média \pm desvio padrão (DP) e variáveis não paramétricas foram expressas em mediana e intervalo interquartil. Os dados categóricos foram descritos em valores absolutos e porcentagem da amostra total. As variáveis paramétricas dos grupos de $\mathrm{AO}$ e $\mathrm{AE}$ foram comparadas pelo teste $\mathrm{t}$ de Student independente e as variáveis não paramétricas foram comparadas pelo teste de MannWhitney. A variáveis categóricas foram analisadas pelo teste do qui-quadrado e a correlação de Pearson usada para analisar a correlação entre variáveis de fatores de risco tais como CC e pressão arterial, bem como porcentagem da DMF. Valores de probabilidade menores que $\mathrm{p}<0,05$ foram considerados estatisticamente significativos. 


\section{Resultados}

Inicialmente recrutamos 56 adolescentes, 26 deles foram excluídos - nove eram classificados como escala de Tanner I, II ou III; cinco estavam em sobrepeso; cinco apresentavam distúrbios endócrinos; um usava medicamento e seis recusaram a participar do estudo. Nossa amostra final foi composta de 30 adolescentes. Assim, estudamos 20 AO (10 do sexo masculino) e $10 \mathrm{AE}$ (5 do sexo masculino) (Figura 1). Sete adolescentes do grupo AO apresentavam SM (35\%) e nenhum AE apresentava SM (Figura 2).

Na Tabela 1, descrevamos as medidas antropométricas e de composição corporal dos participantes. Ambos os grupos foram similares na distribuição de sexo e de idade. Como esperado, AO apresentavam maior peso corporal, IMC, CP e CC. Em relação à composição corporal, observou-se menor porcentagem de água corporal e massa magra e maior massa gorda no grupo de $\mathrm{AO}$.

Dados sobre fatores de risco cardiovasculares nos grupos AO e AE são apresentados na Tabela 2. Não houve diferença nos níveis de HDL ou de glicemia entre os grupos. Em comparação aos $A E$, os $A O$ apresentaram maior PAS e maior PAD, além de níveis maiores de TG. LDL-c, TG/HDL-c, colesterol não HDL, LDL/HDL-c, e colesterol total. No TECP, o grupo de AO apresentou menor $\mathrm{VO}_{2}$ pico em comparação aos AE. Resultados da polissonografia revelaram que um $\mathrm{O}_{2}$ mínimo mais baixo AO em comparação a AE. Não houve diferença no índice de despertares ou no IAH entre os grupos (Tabela 2). No entanto, a maioria dos adolescentes do grupo $\mathrm{AO}(86,6 \%)$ e $50 \%$ dos $\mathrm{AE}$ apresentaram $\mathrm{IAH} \geq 1$ evento/h (Figura 2).
Na Figura 3, apresentamos a prevalência dos fatores de risco para SM segundo a IDF. ${ }^{15} \mathrm{Na}$ Figura $4 \mathrm{~A}$, apresentamos as análises da DMF. Em um participante do grupo AO, foi detectada bifurcação da artéria braquial, e decidimos excluir esse dado das análises. As análises da DMF mostraram que os AO reatividade vascular mais baixa das grandes artérias em comparação ao grupo $\mathrm{AE}(6,17 \pm 2,72 \%$ vs. 9,37 $\pm 2,20 \%$, $p=0,005)$. Diante disso, exploramos a associação entre DFM e os fatores de risco para SM, e encontramos uma associação entre CC e DMF ( $R=-0,506, p=0,008$; Figura 4B), e entre PAS e DMF $(R=-0,493, p=0,006$; Figura $C)$.

\section{Discussão}

O principal achado do presente estudo é o fato de que AO apresentam disfunção endotelial, indicada por uma reatividade vascular reduzida. Além disso, com base nas análises de correlação, podemos sugerir que a CC e os níveis de PAS podem ser preditores desta disfunção.

A obesidade e a SM aumentam o risco de disfunção endotelial, e a SAOS contribui para esse agravamento. Contudo, apesar da alta prevalência de SAOS em AO, não houve diferenças na presença de SAOS e de IAH entre os grupos estudados. Podemos sugerir que, em adolescentes, outros fatores além da obesidade, tais como rinite alérgica, asma e hipertrofia adenotonsilar, possam contribuir com a SAOS. Esses achados foram encontrados em outros estudos. ${ }^{29}$ Assim, no presente estudo, a DMF deficiente não esteve associada à SAOS, e a presença de SAOS não pôde ser atribuída à obesidade ou à SM. Nós especulamos que a

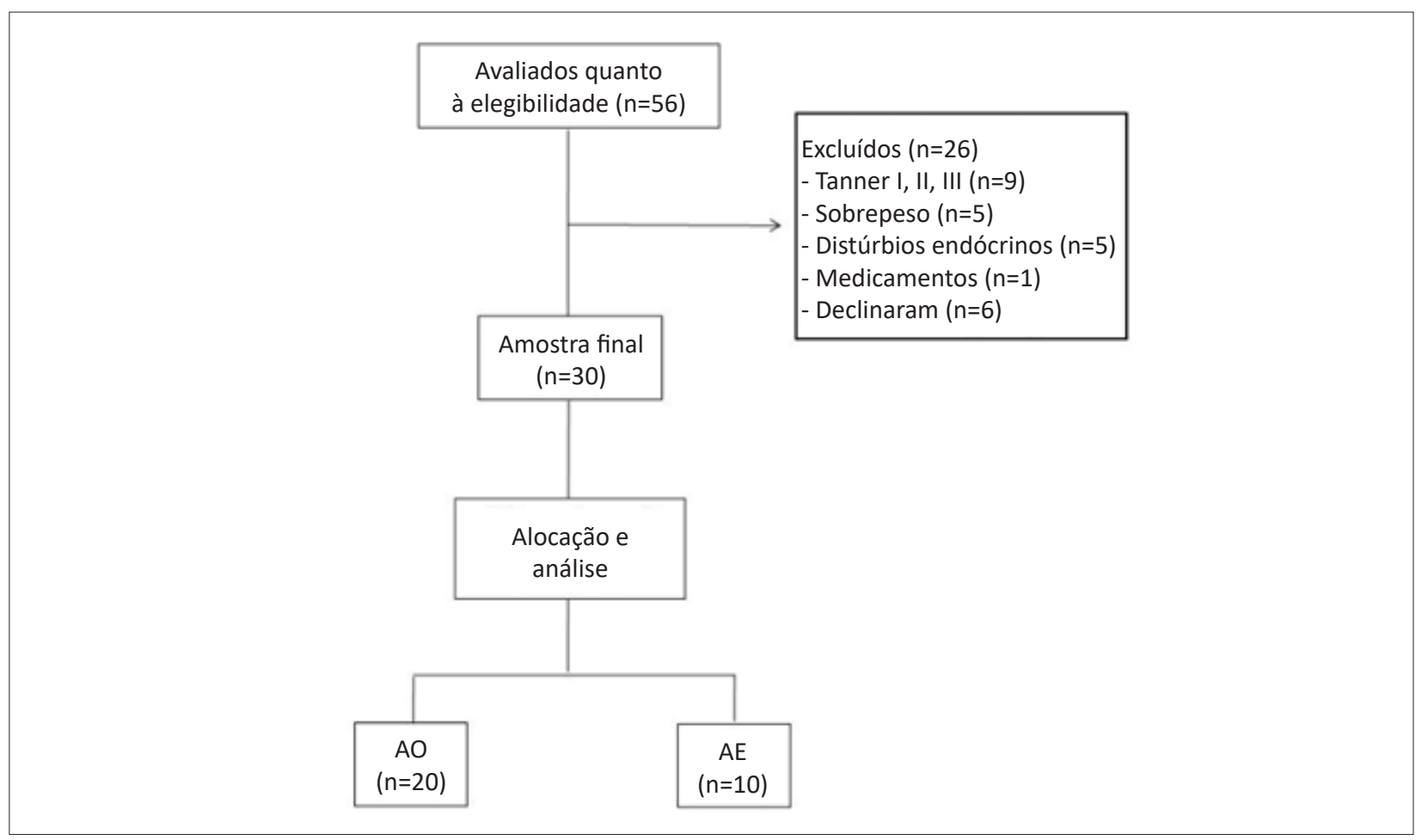

Figura 1 - Fluxograma de inclusão dos participantes no estudo; AO: adolescentes obesos; AE: adolescentes eutróficos. 


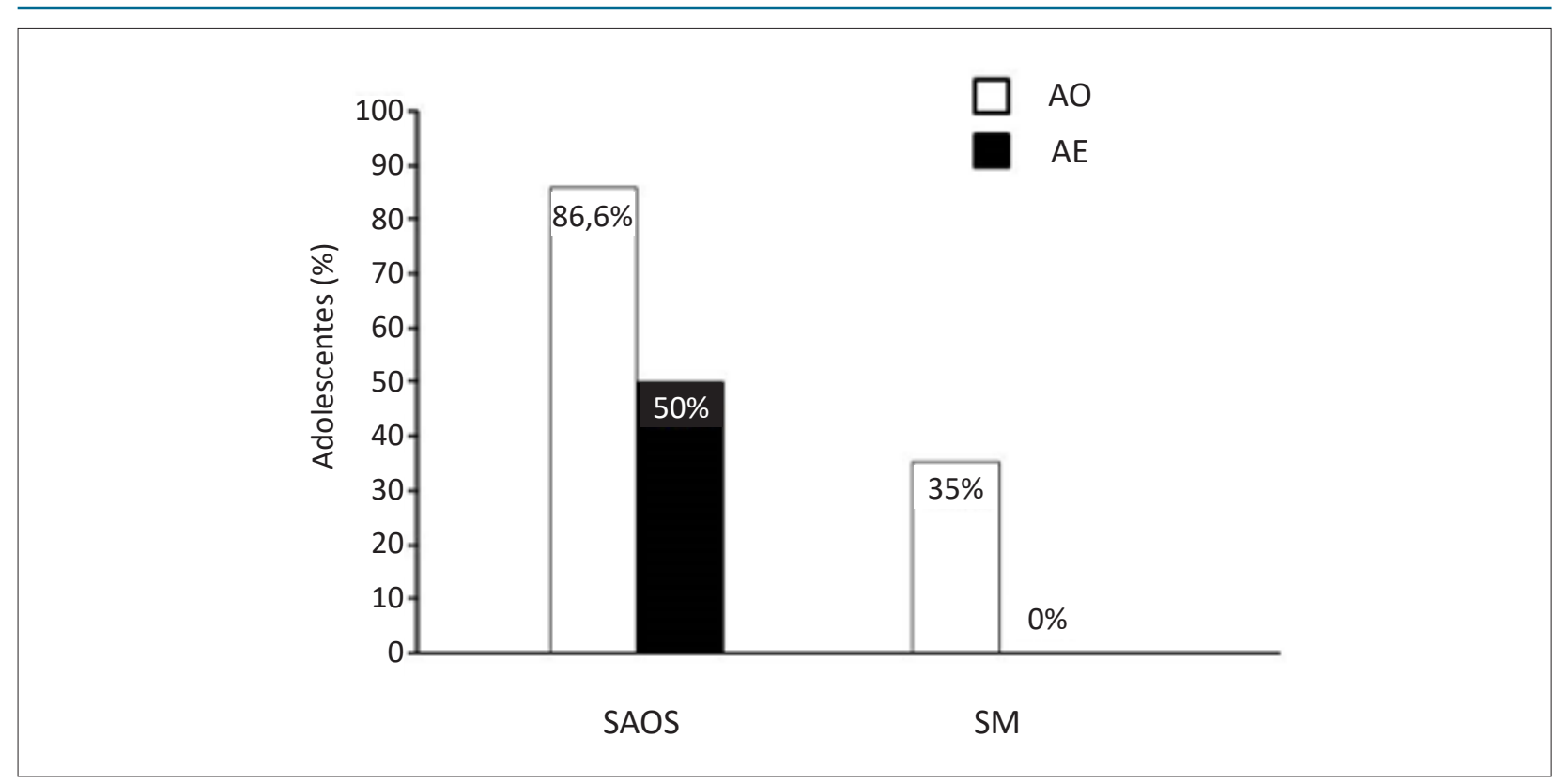

Figura 2 - Porcentagem de síndrome da apneia obstrutiva do sono (SAOS) e síndrome metabólica (SM) em adolescentes obesos (AO) e adolescentes eutróficos (AE).

Tabela 1 - Dados antropométricos e de composição corporal em adolescentes obesos e eutróficos

\begin{tabular}{|c|c|c|c|}
\hline & $A O(n=20)$ & $\operatorname{AE}(n=10)$ & p \\
\hline $\operatorname{Sexo}(M / F)$ & $10 / 10$ & $5 / 5$ & 1 \\
\hline Idade (anos) & $14,2 \pm 1,6$ & $15,2 \pm 1,2$ & 0,075 \\
\hline Peso (kg) & $100,1 \pm 20,3$ & $54,4 \pm 5,3$ & $<0,001$ \\
\hline Altura (m) & $1,67 \pm 0,08$ & $1,65 \pm 0,6$ & 0,760 \\
\hline IMC $\left(\mathrm{kg} / \mathrm{m}^{2}\right)$ & $35,9 \pm 6,2$ & $19,9 \pm 1,8$ & $<0,001$ \\
\hline $\mathrm{CP}(\mathrm{cm})$ & $38,3 \pm 3,6$ & $31,9 \pm 1,8$ & $<0,001$ \\
\hline $\mathrm{CC}(\mathrm{cm})$ & $107,9[100-114,5]$ & $67,5[66,4-73,7]$ & $<0,001$ \\
\hline Água corporal (\%) & $45,4 \pm 3,8$ & $56,5 \pm 4,6$ & $<0,001$ \\
\hline Gordura corporal (\%) & $38 \pm 5,2$ & $22,9 \pm 6,3$ & $<0,001$ \\
\hline Massa magra (\%) & $62 \pm 5,2$ & $77,1 \pm 6,3$ & $<0,001$ \\
\hline
\end{tabular}

Dados paramétricos apresentados em média \pm desvio padrão. Dados não paramétricos apresentados em mediana e intervalo interquartil. AO: adolescentes obesos; AE: adolescentes eutróficos; IMC: índice de massa corporal; CP: circunferência do pescoço; CC: circunferência da cintura.

SM potencializou a disfunção endotelial no grupo de $\mathrm{AO}$, uma vez que $35 \%$ dos pacientes obesos na nossa amostra apresentaram SM.

A aterosclerose e as manifestações clínicas da DCV originam-se na infância, ${ }^{30}$ e sua detecção precoce é muito importante para sua prevenção. A disfunção endotelial é considerada um sinal precoce de aterosclerose em crianças com fatores de risco para DCV, e pode ser revertida por intervenções que visam diminuir o risco cardiovascular. ${ }^{30}$
A DMF com hiperemia reativa é um método não invasivo que avalia a vasodilatação dependente de endotélio, mediada por óxido nítrico (NO), e é um método diagnóstico adequado para o grupo etário estudado. Uma meta-análise identificou que um aumento em $1 \%$ na DMF aumenta o risco futuro de eventos cardiovasculares em $13 \%{ }^{3}$ Há evidência de que crianças e adolescentes obesos apresentam menor complacência e distensibilidade vascular em comparação àqueles com peso normal. ${ }^{3}$ Tal fato poderia explicar os níveis mais elevados de pressão sanguínea nos $\mathrm{AO} .{ }^{31}$ 
Artigo Original

Tabela 2 - Fatores de risco cardiovascular em adolescentes obesos e eutróficos

\begin{tabular}{|c|c|c|c|}
\hline & $O A(n=20)$ & $E A(n=10)$ & $\mathbf{p}$ \\
\hline PAS (mmHg) & $120[110-127,5]$ & $110[100-110]$ & 0,001 \\
\hline PAD (mmHg) & 75 [70-80] & $65[60-70]$ & 0,005 \\
\hline Glicemia (mg/mL) & $84,9 \pm 5,4$ & $89,3 \pm 7,2$ & 0,140 \\
\hline TG (mg/dL) & $120,5 \pm 48,3$ & $71,1 \pm 28,8$ & 0,020 \\
\hline $\mathrm{HDL}-\mathrm{c}(\mathrm{mg} / \mathrm{dL})$ & $41,2 \pm 7,7$ & $48,4 \pm 10,7$ & 0,079 \\
\hline TG/HDL-c ratio & $3,1 \pm 1,6$ & $1,6 \pm 1$ & 0,011 \\
\hline LDL-c (mg/dL) & $97,5 \pm 25,7$ & $69,9 \pm 22,2$ & 0,015 \\
\hline nHDL-c (mg/dL) & $121,5 \pm 27,5$ & $83,2 \pm 26,2$ & 0,004 \\
\hline LDL/HDL-c radio & $2,4 \pm 0,8$ & $2,6 \pm 0,7$ & 0,007 \\
\hline Colesterol total (mg/dL) & $162,7 \pm 28,7$ & $132,5 \pm 24,1$ & 0,016 \\
\hline \multicolumn{4}{|c|}{ Polissonografia de noite inteira } \\
\hline IAH (events/h) & $5,6 \pm 3,8$ & $3,1 \pm 3,4$ & 0,121 \\
\hline $\mathrm{O}_{2}$ Sat mínimo (\%) & 90 [81-90] & $92,5[88,5-93]$ & 0,026 \\
\hline Índice de despertares & $50,6 \pm 18,1$ & $50 \pm 9,3$ & 0,943 \\
\hline \multicolumn{4}{|c|}{ Teste de esforço cardiopulmonar } \\
\hline $\mathrm{VO}_{2}$ pico (mL/kg/min) & $30,6 \pm 7,7$ & $23,4 \pm 5,9$ & 0,022 \\
\hline
\end{tabular}

Dados paramétricos apresentados em média $\pm D P$. Dados não paramétricos expressos em mediana e intervalo interquartil. AO: adolescentes obesos; AE: adolescentes eutróficos; PAS: pressão arterial sistólica; PAD: pressão arterial diastólica; TG: triglicerídeos; HDL: lipoproteína de alta densidade; LDL: lipoproteína de baixa densidade.

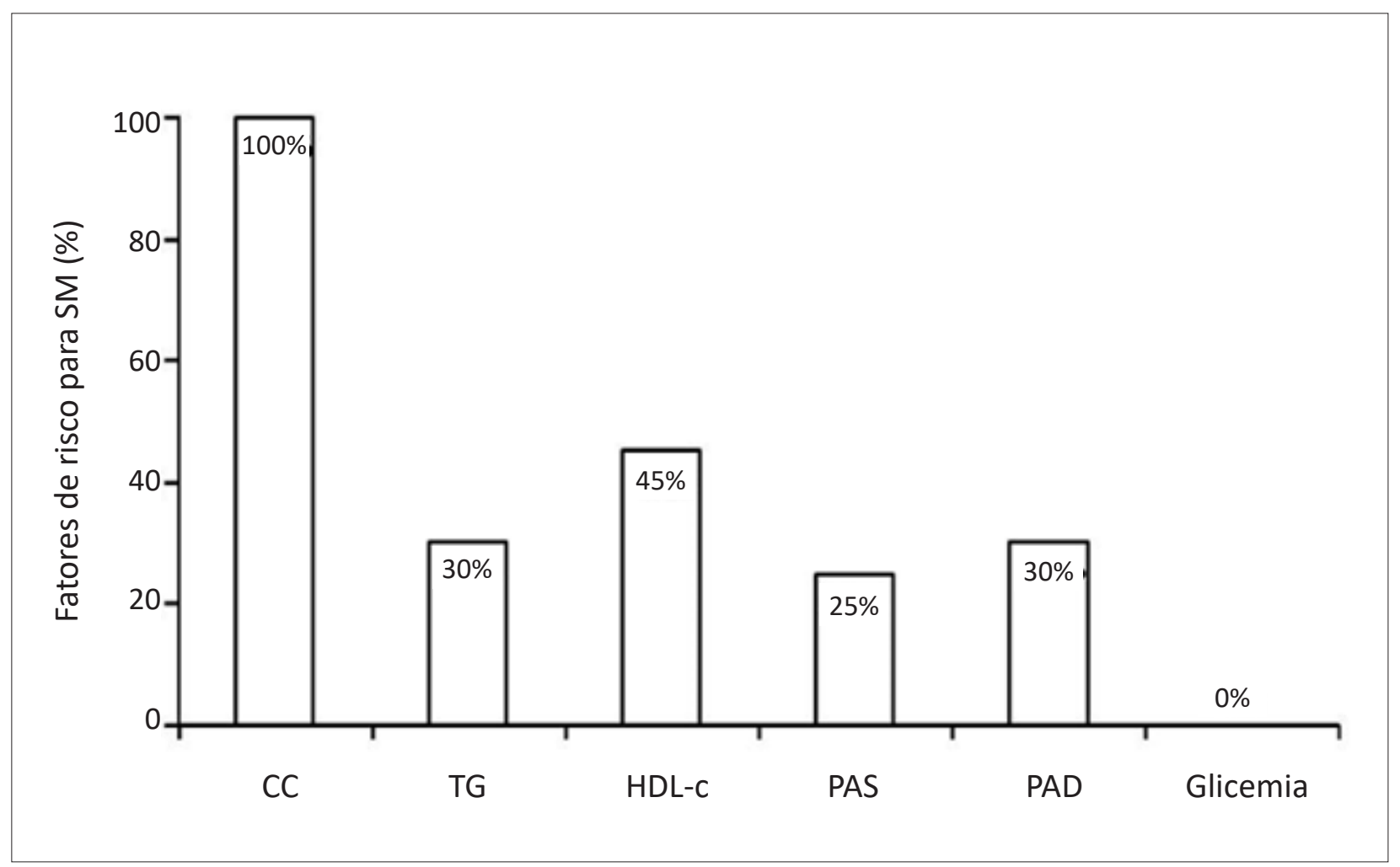

Figura 3 - Porcentagem de fatores de risco para sindrome metabólica (SM) em adolescentes obesos; CC: circunferência da cintura; TG: triglicerídeos; HDL: lipoproteína de alta densidade; PAS: pressão arterial sistólica; PAD: pressão arterial diastólica. 


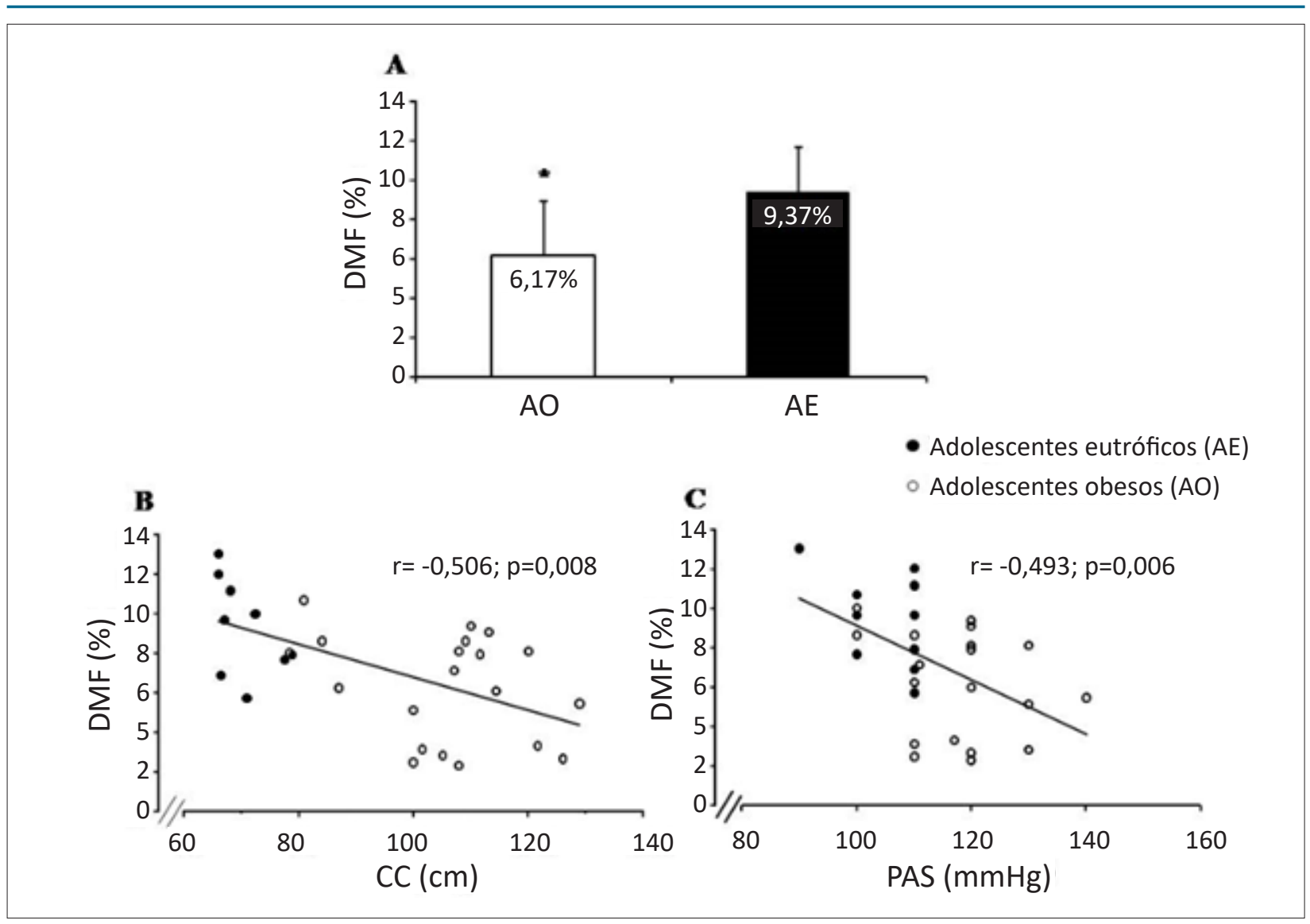

Figura 4 - Hiperemia reativa por dilatação mediada pelo fluxo (DMF) (A); coeficiente de correlação entre DMF e circunferência da cintura (CC) (B); e coeficiente de correlação entre DMF e pressão arterial sistólica (PAS) em adolescentes obesos (AO) e adolescentes eutróficos (AE) (C). * $p<0,005$.

Um estudo conduzido com adultos relatou uma DMF de $9,4 \% \pm 4,7 \%$. $^{32}$ Uma meta-análise de Dias et al., ${ }^{3}$ identificou uma DMF de $6,0 \% \pm 0,69 \%$ em adolescentes obesos, em comparação a $12,32 \% \pm 3,14 \%$ em adolescentes eutróficos. ${ }^{3}$ Esse dado corrobora nossos achados, que mostraram uma reatividade vascular reduzida em $\mathrm{AO}$ em comparação a $\mathrm{AE}$ $(6,17 \pm 2,72 \%$ e $9,37 \pm 2,20 \%$, respectivamente)

A DMF é uma medida indireta da biodisponibilidade do $\mathrm{NO}, 26,28,32$ uma vez que ela estimula um meio isquêmico e, em seguida, vasodilatação. A oclusão dos vasos leva à liberação de adenosina, fator hiperpolarizante derivado do endotélio, íons de hidrogênio, entre outras substâncias, com o objetivo de restaurar a perfusão sanguínea via dilatação da microcirculação. Neste método, quando o manguito é esvaziado, a circulação é restaurada com aumento do aporte sanguíneo para a região isquêmica, causando "hiperemia reativa". A tensão de cisalhamento, causada pelo aumento do fluxo sanguíneo, e sua velocidade, leva à liberação de substâncias vasodilatadoras pelo endotélio, tais como o NO, via ativação da enzima óxido nítrico sintase endotelial (eNOS), e consequente relaxamento da musculatura lisa vascular e aumento no diâmetro arterial. Uma capacidade de relaxamento mais baixa leva à disfunção endotelial. ${ }^{26,28,32}$ Uma menor vasodilatação pode ocorrer nos meninos em comparação às meninas, além de existir uma variação de função endotelial durante o ciclo menstrual. ${ }^{28}$
Portanto, nós realizamos os exames na primeira fase do período menstrual. O EndoPAT ${ }^{\circledR}$ foi avaliado por Radke et al. em relação aos estágios da puberdade. ${ }^{33} \mathrm{Um}$ IHR mais baixo foi observado na puberdade em comparação aos estágios de Tanner 4 e 5, com variação entre 1,11 e 1,70. O ponto de corte para adultos é 1,35, o que poderia ser usado para identificar os indivíduos com disfunção endotelial na microcirculação. Essa técnica foi desenvolvida para ser independente de examinador. Sabe-se que, devido à localização do manguito a ser inflado, a vasodilatação da microcirculação obtida não é totalmente dependente de NO. Assim, enquanto o EndoPAT ${ }^{\circledR}$ mede a função endotelial da microcirculação, e DMF avalia a função endotelial das artérias condutoras. É possível que os resultados sejam comparativamente discrepantes, porém complementares, uma vez que os métodos avaliam sistemas diferentes. ${ }^{28}$ Nos adolescentes avaliados neste estudo, não houve diferença nesta medida entre $\mathrm{AO}$ e $\mathrm{AE}$ e, assim, não conseguimos identificar complementariedade entre EndoPAT ${ }^{\circledR}$ e DMF.

Outro fator relevante no estudo foi a correlação entre CC e reatividade vascular. Uma CC aumentada é um preditor de risco para DCV, ${ }^{15,34}$ conhecido como "síndrome da adiposidade visceral". ${ }^{4,34}$ Com o aumento da adiposidade visceral, ocorre um aumento nos depósitos de gordura patogênica e piora na reatividade vascular. A distribuição da gordura visceral é um fator preditivo de hipertensão, maior que o aumento 
generalizado em gordura. O sistema nervoso simpático parece estar relacionado a diferentes componentes da síndrome da adiposidade a visceral, gerando um aumento real na atividade simpática, ${ }^{34}$ e um risco aumentado de hipertensão nesses pacientes.

Apesar de o IAH não ter sido diferente entre os dois grupos estudados, houve uma prevalência de $86,6 \%$ e $50 \%$ de SAOS e de $35 \%$ e $0 \%$ de $S M$ nos grupos $\mathrm{AO}$ e $\mathrm{AE}$, respectivamente. A maior presença de SAOS e SM pode ter contribuído para o aumento de PAS nesse grupo, o que pode ter sido modulado por um tônus simpático aumentado. Esse fato já foi observado por Trombetta et al., que relataram maior atividade e barorreflexo reduzido em pacientes adultos com SM associada à SAOS. ${ }^{10}$

No presente estudo, observamos que $\mathrm{AO}$ exibiram $\mathrm{VO}_{2}$ pico reduzido, sugerindo um risco cardiovascular aumentado. De fato, existe forte evidência de que a obesidade associa-se com um pior prognóstico em adolescentes com capacidade funcional reduzida e presença de comorbidades cardiometabólicas. Medidas preventivas são necessárias nesses indivíduos com disfunção endotelial, estimulando a prática de atividade física e dieta saudável visando a redução da CC e da pressão arterial.

\section{Limitações}

Nosso estudo tem várias limitações. Primeiro, considerando que, nas meninas, existe variação da função endotelial durante o ciclo menstrua ${ }^{28}$ e, apesar de termos realizado o teste na primeira fase do ciclo menstrual, algumas tiveram apenas a menarca e, portanto, não apresentavam regularidade ou conhecimento do ciclo. Segundo, uma vez que não existe consenso sobre os critérios diagnósticos para SAOS em indivíduos com idade entre 13 e 18 anos, no presente estudo, similar a outros, ${ }^{35,36}$ utilizamos valores pediátricos. Os critérios usados para a idade de até 13 anos foram estendidos para até 18 anos, com base no manual da AASM para pontuação de sono e eventos associados. ${ }^{23}$

\section{Conclusão}

$\mathrm{Na}$ amostra estudada, a obesidade foi um importante fator de risco para o desenvolvimento de SM, e levou à disfunção endotelial, a qual é o ponto inicial da formação da placa de ateroma. Além disso, CC e PAS aumentadas são preditoras de disfunção endotelial em adolescentes. A SAOS estava presente na maioria dos adolescentes, independentemente da obesidade.

\section{Referências}

1. World Health Organization. (WHO). Ending Childhood Obesity. Geneva;2016.

2. Brasil. Ministério da Saúde, Instituto Brasileiro de Geografa e Estatística. (IBGE). Pesquisa Nacional de Saúde do Escolar 2015. Brasília-DF:IBGE; 2016. $132 \mathrm{p}$.

3. Dias KA, Green DJ, Ingul CB, Pavey TG, Coombes J.S. Exercise and Vascular Function in Child Obesity: A Meta-Analysis. Pediatrics. 2015,136(3), e648e659.

\section{Agradecimentos}

Os autores desejam agradecer a todos os participantes do estudo. Agradecimento especial à equipe do Departamento de Reabilitação Cardiopulmonar da Universidade Nove de Julho (UNINOVE), particularmente à Profa Dra. Simone Dal Corso pelo apoio logístico e auxílio com o Teste de Esforço Cardiopulmonar (TECP)

Este trabalho foi financiado pela Universidade Nove de Julho (UNINOVE), São Paulo, Brasil. MFH foi apoiada pela Universidade Nove de Julho (UNINOVE), FXC foi apoiado pela FAPESP (\# 2015 / 03274-0 e \# 2016 / 16831-7) e Coordenação de Aconselhamento de Pessoal de Nível Superior (CAPES). RRPL-V, LV e KYK foram apoiadas pela Coordenação de Aconselhamento de Pessoal de Nível Superior (CAPES). EFO e FMCC foram apoiados pela FAPESP (\#15/11738-6 para EFO). ICT e LVFO foram apoiados pelo Conselho Nacional de Pesquisa (CNPq \# 302809 / 2018-0 para ICT, CNPq \#313053/2014-6 para LVFO, respectivamente). As agências financiadoras não tiveram nenhum papel no desenho do estudo, coleta e análise de dados, decisão de publicar ou preparação do manuscrito.

\section{Contribuição dos Autores}

Concepção e desenho da pesquisa: Hussid MF, Cepeda FX, Consolim-Colombo FM, Trombetta IC; Obtenção de dados: Jordão CP, Lopes-Vicente RRP, Virmondes L, Katayama KY, Oliveira EF, Oliveira LVF; Análise e interpretação dos dados: Hussid MF, Cepeda FX, Oliveira LVF, Trombetta IC; Análise estatística, Redação do manuscrito e Revisão crítica do manuscrito quanto ao conteúdo intelectual importante: Hussid MF, Cepeda FX, Trombetta IC.

\section{Potencial Conflito de Interesses}

Declaro não haver conflito de interesses pertinentes.

\section{Fontes de Financiamento}

O presente estudo não teve fontes de financiamento externas.

\section{Vinculação Acadêmica}

Este artigo é parte de dissertação de Mestrado de Maria Fernanda Hussid pela Universidade Nove de Julho - UNINOVE.

4. Mongraw-Chaffin, M, Foster MC, Kalyani RR, Vaidya D, Burke GL, Woodward $M$,et al. Obesity severity and duration are associated with incident metabolic syndrome: Evidence against metabolically healthy obesity from the multi-ethnic study of atherosclerosis. J. Clin. Endocrinol. Metab. 2016, 101(11), 4117-24.

5. Cristina D, Masquio L, Ganen ADP, Munhoz R, Sanches PDL, Corgosinho FC, et al. Cut-off values of waist circumference to predict metabolic syndrome in obese adolescents. Nutr Hosp. 2015; 31(4):1540-50. 
6. Vanhoutte PM. Endothelial dysfunction: a multifaceted disorder. Am J Physiol Hear Circ Physiol. 2006;291(3):H985-H1002.

7. Thijssen DH, Black MA, Pyke KE, Padilla J, Atkinson G, Harris RA, et al. Assessment of flow-mediated dilation in humans: a methodological and physiological guideline. Am J Physiol Heart Circ Physiol. 2011;300(1); H2-12.

8. Gileles-Hillel A, Alonso-Alvarez ML, Kheirandish-Gozal L, Peris E, CorderoGuevara JA,et al. Inflammatory markers and obstructive sleep apnea in obese children: the NANOS study. Mediat Inflamm. 2014; 2014:1-9.605280

9. Bhushan B, Ayub B, Loghmanee DA, Billings KR. Metabolic alterations in adolescentes with obstructive sleep apnea. Int J Pediatr Otorhinolaryngol. 2015;79(12):2368-73.

10. Trombetta IC, Somers VK, Maki-Nunes C, Drager LF. Syndrome — Implications for Cardiovascular Risk. Sleep.2010;33(9):1193-9.

11. Bhattacharjee R, Kim J, Alotaibi WH, Kheirandish-Gozal L, Capdevila OS, Gozal D. Endothelial dysfunction in children without hypertension: Potential contributions of obesity and obstructive sleep apnea. Chest. 2012, 141(3):682-91.

12. Brasil.Ministério da Saúde. Orientações para o atendimento à saúde do adolescente. Brasília, DF; 2008.

13. Brasil. Ministério da Saúde. Orientações para o atendimento à saúde do adolescente. Brasília, DF; 2008.

4. World Health Organization. (WHO).The WHO Child Growth Standards. Geneva; 2023.

15. Consensus Statements - IDF Consensus definition of the metabolic in children. (Acessed in 2019 Jun 12).Available from: idf.org/e-library/consensusstatement/61-idf-consensus-definition.html

16. Fernandez JR, Redden DT, Pietrobelli A, Allison DB. Waist circumference percentiles in nationally representative samples of african-american, europeanamerican, and mexican-american children and adolescents. J Pediatr.2004; 145(4):439-44.

17. Coutinho CA, Longui CA, Monte O,CondeW, Kochi C. Measurement of neck circumference and its correlation with body composition in a sample of students in São Paulo, Brazil. Horm Res Paediatr. 2014, 82(3):179-86.

18. Brasil.Ministério da Saúde. Secretaria de Atenção à Saúde. Departamento de Atenção Básica. Hipertensão arterial sistêmica para o Sistema Único de Saúde. Brasília (DF); 2006. (citado em 201920 jan). Available from: http://dab.saude. gov.br/docs/publicacoes/cadernos_ab/abcad15.pdf

19. Urbina E, Alpert, B, Flynn J, Hayman L, Harshfield GA, Jacobson M, et al. Ambulatory Blood Pressure Monitoring in Children and Adolescents : Recommendations for Standard Assessment A Scientific Statement From the American Heart Association Atherosclerosis, Hypertension, and Obesity in Committee of the Council on Cardiovascular Disease in the Young and the Council for High. Hypertension. 2008;52(3):433-51.

20. Nobre F, Saad CI R, Giorgi DM, Mion D, Sociedade Brasileira de Cardiologia, Sociedade Brassileira de Hipertensão, Sociedade Brasileira de Nefrologia. VI Diretrizes Brasileiras. Arq Bras Cardiol. 2010;95:1-51,

21. The Report of an American Academy of Sleep Medicine Task Force. Sleep related breathing disorders in adults: recommendations for syndrome definition and measurement techniques in clinical research. Sleep. 1999;22(5):667-89.
22. Iber C, Ancoli-Israel S, Chesson A, Quan SF. The AASM manual for the scoring of sleep and associated events: Rules, terminology and technical specifications.American Academy of Sleep Medicine;2007.

23. Berry, R.B.; Budhiraja, R.; Gottlieb, D.J.; Gozal, D.; Iber, C.; Kapur, V.K.; Marcus, C.L.; 23.Mehra, R.; Parthasarathy, S.; Quan, S.F.; et al. Rules for Scoring Respiratory Events in Sleep : Update of the 2007 AASM Manual for the Scoring of Sleep and Associated Events. J Clin Sleep Med. 2012;8(5):597-619.

24. Neder JA, Nery LE. Teste de exercício cardiopulmonar. J Pneumol .2002, 28(Supl 3):166-206.

25. Neder JA, Nery LE, Castelo A, Andreoni S, Lerario MC, Sachs A et al. Prediction of metabolic and cardiopulmonary responses to maximum cycle ergometry: a randomised study. Eur Respir J. 1999;14(6):1304-13.

26. Celermajer DS, Sorensen KE, Gooch VM, Spiegelhalter DJ, Miller O, Sullivan ID et al. Noninvasive detection of endothelial dysfunction in children and adults at risk of atherosclerosis. Lancet. 1992;340(8828):111-5

27. Bonetti PO, Pumper GM, Higano ST, Holmes DR, Kuvin JT, Lerman A. Noninvasive identification of patients with early coronary atherosclerosis by assessment of digital reactive hyperemia. J. Am. Coll. Cardiol. 2004;44(11):2137-41.

28. Bruyndonckx L, Hoymans VY, Van Craenenbroeck AH, Vissers DK, Vrints CJ, Ramet J, et al. Assessment of Endothelial Dysfunction in Childhood Obesity and Clinical Use. Oxid Med Cell Longev. 2013, 2013: 1-19. 174872.

29. Redline S, Tishler P V, Schluchter M, Aylor J, Clark K, Graham G. Risk Factors for Sleep-disordered Breathing in Children Associations with Obesity, Race, and Respiratory Problems African Americans appears to be independent of the effects of obesity or respiratory problems . Red-. Am J Respir Crit Care Med. 1999;159(5Pt1): 1527-32.

30. Hopkins ND, Dengel DR, Green DJ, Age and sex relationship with flowmediated dilation in healthy children and adolescents. J. Appl. Physiol. 2015;119(8):926-33.

31. Wilk G, Osmenda G, Matusik P, Nowakowski D, Jasiewicz-Honkisz $B$, Ignacak A, et al.Endothelial function assessment in atherosclerosis: Comparison of brachial artery flow-mediated vasodilation and peripheral arterial tonometry. Pol Arch Med Wewn. 2013;123(9):443-52.

32. Consolim-Colombo, F.M.; Costa-Hong V., Katayama K.Y. Método de investigação da função endotelial em humanos. In: Endotélio e Doenças Cardiovasculares,São Paulo:Atheneu; 2016.

33. Radtke T, Khattab K, Eser P, Kriemler S, Saner H, Wilhelm M. Puberty and microvascular function in healthy children and adolescents. J Pediatr. 2012; 161(5):887-91.

34. Lopes HF, Correa Gianella ML, Consolim Colombo FM, Egan BM, Visceral adiposity syndrome .Diabetol Metab Syndr. 2016 Jul 1;8:40.

35. Oliveira, V.X.N.; Teng, A.Y. The Clinical Usefulness of Sleep Studies in Children. Paediatr. Respir Rev. 2016;17:53-6.

36. Marcus CL, Brooks LJ, Ward SD, Draper KA, Gozal D, Halbower AC, et al. The clinical usefulness of sleep studies in children.Diagnosis and Management of Childhood Obstructive Sleep Apnea Syndrome. Pediatrics. 2012;130(3):e714-e755. 